\title{
Factores de riesgo asociados a las complicaciones y a la falla terapéutica en las reconstrucciones de lesiones de vía biliar secundarias a colecistectomía
}

\section{Surgical outcome and risk factors of bile duct injury repair following cholecystectomy}

\author{
Gustavo Martínez-Mier ${ }^{1,2 *}$, Héctor J. Luna-Ortiz³, Nicolás Hernández-Herrera ${ }^{3}$, Stefan Zilli-Hernandez² y \\ Francisco A. Lajud-Barquin ${ }^{2}$ \\ ${ }^{1}$ Cirugía de Trasplantes y Hepatobiliar; ${ }^{2}$ Departamento de Investigación; ${ }^{3}$ Departamento de Cirugía General. Unidad Médica de Alta Especialidad \\ Hospital de Especialidades 14 Adolfo Ruiz Cortines, Instituto Mexicano del Seguro Social, Veracruz, México
}

\section{Resumen}

Antecedentes: Las lesiones de vías biliares por colecistectomía son una complicación seria. Numerosos factores alteran el resultado de su reconstrucción. Método: Evaluación de la reconstrucción de lesiones de vía biliar y sus factores de riesgo (de enero de 2008 a enero de 2017). Resultados: Se evaluaron 58 pacientes (72.4\% mujeres; media de edad 41.8 años). El $67.8 \%$ tuvo colecistectomía laparoscópica como cirugía inicial. El 79.3\% se diagnosticó posoperatoriamente. La referencia promedio fue 9 semanas y su reparación temprana (< 1 semana) ocurrió en el 27.6\%. La lesión más común fue Amsterdam tipo D (55.2\%) y Bismuth-Strasberg E4 (34.5\%). La morbilidad fue del 39.6\% (fuga biliar 10.3\%, estenosis 6.9\% y colangitis recurrente12.1\%), con un $3 \%$ de mortalidad perioperatoria. Se logró un éxito del $81 \%$ de acuerdo con la clasificación de McDonald. Los factores de riesgo para las complicaciones fueron baja hemoglobina, baja albúmina, baja fosfatasa alcalina, tutores biliares y lesiones E3-E5 (análisis univariado). Los factores de riesgo para falla terapéutica fueron la reparación previa en otro centro, los tutores biliares y la reparación posterior a 1 semana tras la lesión (univariado y multivariado). Conclusión: Pueden obtenerse buenos resultados en las reconstrucciones de vías biliares en centros especializados. Existen algunos factores de riesgo para los resultados de las reconstrucciones que deben de ser validados.

PALABRAS CLAVE: Lesión de vía biliar. Reconstrucción quirúrgica. Hepaticoyeyunostomía. Complicaciones. Resultados.

\begin{abstract}
Background: Bile duct injury during cholecystectomy is a serious complication. Multiple factors may alter their outcome. Method: We retrospectively evaluated our results following bile duct injury surgery repair and possible poor outcome risk factors from January 2008 to January 2017. Results: 58 patients (72.4\% female; mean age 41.8 years) were evaluated. $67.8 \%$ underwent open cholecystectomy as initial surgery. $79.3 \%$ of bile duct injury were diagnosed postoperatively. Mean referral time was 9 weeks and early (< 1 week) repair was performed in 27.6\%. Most common lesion was Amsterdam type D (55.2\%) and Bismuth-Strasberg E4 (34.5\%). Morbidity was 39.6\%. Biliary leak occurred in $10.3 \%$, bilio-enteric stricture in $6.9 \%$ and recurrent cholangitis in $12.1 \%$, with $3 \%$ perioperative mortality. There was an $81 \%$ treatment success rate (McDonald classification). Risk factors for complications were: low hemoglobin, low albumin, low alkaline phosphatase, biliary stents and E-3-E5 lesions (univariate analysis only). Risk factors for treatment failure were: previous repair outside our center, use of biliary stents
\end{abstract}

\author{
Correspondencia: \\ *Gustavo Martínez-Mier \\ Corporativo San Gabriel, Alacio Pérez No. 928 Cons. 314, \\ Col. Zaragoza Fecha de recepción: 21-03-2018 \\ C.P. 91910, Veracruz, México Fecha de aceptación: 11-07-2018 \\ E-mail: gmtzmier@gmail.com; $\quad$ DOI: 10.24875/CIRU.18000276
}

Cir Cir. 2018;86:491-498

gustavo.martinezmi@imss.gob.mx

Contents available at PubMed
www.cirugiaycirujanos.com 
and repair later than 1 week after lesion (univariate and multivariate analysis). Conclusions: Good efficacy and safety outcomes in bile repair surgery can be achieved in specialized centers. There are possible risk factors influencing outcomes that should be further validated.

KEY WORDS: Bile duct injury. Cholecystectomy. Surgical repair. Hepatojejunostomy. Complications. Outcome.

\section{Introducción}

Las lesiones de vía biliar (LVB) secundarias a una colecistectomía son complicaciones serias y complejas. La incidencia de LVB posterior a una colecistectomía, sea convencional (CC) o laparoscópica (CL), varía del 0.3 al $1.4 \%$, dependiendo del criterio utilizado para la lesión y de la población estudiada ${ }^{1-3}$. Las LVB pueden tener complicaciones a corto y largo plazo, cursar con estancias hospitalarias prolongadas, requerir intervenciones adicionales y alterar la calidad de vida de estos pacientes ${ }^{4,5}$.

Las LVB son un reto quirúrgico y su manejo requiere las habilidades de cirujanos hepatobiliares en centros de referencia ${ }^{6-9}$. El refinamiento de la técnica quirúrgica y los avances en el cuidado posoperatorio han disminuido considerablemente la morbilidad asociada a la reconstrucción de las LVB. No obstante, las complicaciones posoperatorias de las reconstrucciones de las LVB son aún relativamente frecuentes.

Las reconstrucciones de las LVB tienen resultados exitosos que van desde un $75 \%$ hasta un $98 \%$ en grandes centros hospitalarios de referencia ${ }^{10-19}$. Múltiples factores pueden alterar los resultados y las complicaciones de este tipo de procedimientos, los cuales incluyen el tiempo de la reconstrucción, la sepsis asociada, la lesión vascular y el uso de tutores biliares transanastomóticos ${ }^{10-16}$. Los resultados y sus complicaciones también pueden verse afectados si los pacientes fueron referidos tras una reconstrucción previa fuera de un centro de tercer nive ${ }^{17}$, o según el tiempo y el momento del envío ${ }^{10,17-22}$. Debido a lo anterior, hemos decidido analizar nuestros resultados de las reconstrucciones de LVB para identificar los factores asociados a nuestras complicaciones y a su éxito.

\section{Método}

Se realizó una revisión retrospectiva de los expedientes de los pacientes que sufrieron una LVB desde enero de 2008 hasta enero de 2017 referidos a nuestra unidad. El estudio fue previamente aprobado por el comité local de ética e investigación de la unidad. Se incluyeron en el estudio reconstrucciones posteriores a secciones totales o mayores del conducto biliar común, hepático común o conductos segmentarios mayores. También se incluyeron reconstrucciones por estenosis benignas posteriores a una colecistectomía con o sin exploración de vías biliares. Se excluyeron fugas biliares menores del conducto cístico y de la fosa vesicular, así como estenosis secundarias a pancreatitis crónicas y malignidad, litiasis biliar, estenosis del esfínter de Oddi y colangitis esclerosante primaria.

\section{Datos y manejo preoperatorio}

Se registraron los datos demográficos y de laboratorio de los pacientes, además del procedimiento inicial realizado (CC, CL o ambos) y el momento del diagnóstico de la lesión (transoperatorio o posoperatorio). Dentro de los datos a registrar se determinó si el paciente había tenido una reconstrucción previa fuera de la unidad o no. Se registró el tiempo de referencia, y se definió el tiempo de reconstrucción como temprano si esta se realizó durante la primera semana posterior a la LVB o como intermedio si se hizo en menos de 6 semanas después de la LVB. Se registró también si la reconstrucción se llevó a cabo durante la misma admisión de la cirugía inicial.

\section{Clasificación de las LVB}

Las LVB se clasificaron de acuerdo con la clasificación del Amsterdam Academical Medical Center ${ }^{23}$, y el nivel de la lesión se determinó mediante la clasificación combinada Bismuth-Strasberg ${ }^{24,25}$ (Tabla 1).

\section{Manejo quirúrgico}

Los pacientes se sometieron a reconstrucción de las LVB cuando se cumplieron las siguientes condiciones: 1) ausencia de biloma (documentado por técnicas de imagen, sea ultrasonido o tomografía); 2) identificación adecuada de la anatomía biliar mediante colangiografía por resonancia magnética o colangiopancreatografia; y 3) ausencia de sepsis, colangitis o falla orgánica.

El tratamiento quirúrgico estándar consistió en una hepaticoyeyunostomía término-lateral mediante disección cefálica hasta obtener unos conductos biliares de 
Tabla 1. Clasificación de las lesiones de vía biliar

\begin{tabular}{|c|c|c|c|}
\hline \multicolumn{2}{|c|}{ Amsterdam Academical Medical Center } & \multicolumn{2}{|c|}{ Bismuth-Strasberg } \\
\hline Tipo & Descripción & Tipo & Descripción \\
\hline A & $\begin{array}{l}\text { Fugas del conducto cístico o fuga de un conducto hepático } \\
\text { aberrante o periférico }\end{array}$ & $\mathrm{E} 1$ & $\begin{array}{l}\text { División/estenosis del conducto hepático común a más de } 2 \\
\mathrm{~cm} \text { de la bifurcación }\end{array}$ \\
\hline B & $\begin{array}{l}\text { Fuga de un conducto biliar mayor con o sin estenosis biliar } \\
\text { concomitante }\end{array}$ & E2 & $\begin{array}{l}\text { División/estenosis del conducto hepático común a menos de } \\
2 \mathrm{~cm} \text { de la bifurcación }\end{array}$ \\
\hline C & Estenosis de conductos biliares sin fuga biliar & E3 & $\begin{array}{l}\text { División/estenosis del conducto hepático común a nivel de } \\
\text { la bifurcación }\end{array}$ \\
\hline \multirow[t]{2}{*}{ D } & $\begin{array}{l}\text { Transección completa del conducto con o sin escisión de alguna } \\
\text { porción del árbol biliar }\end{array}$ & E4 & $\begin{array}{l}\text { División/estenosis que involucra la confluencia con pérdida } \\
\text { de la comunicación del conducto hepático derecho e } \\
\text { izquierdo }\end{array}$ \\
\hline & & E5 & $\begin{array}{l}\text { Conducto hepático derecho aberrante con estenosis } \\
\text { concomitante del conducto hepático común }\end{array}$ \\
\hline
\end{tabular}

aspecto macroscópicamente sanos. Se dividió la placa hiliar cuando fue necesario. Se realizó anastomosis intrahepáticas mediante resección parcial del segmento IV si no se identificaron conductos biliares extrahepáticos. Todas las anastomosis se realizaron con sutura absorbible monofilamento 4-0 o 5-026. A juicio del cirujano, se colocaron tutores transhepáticos de Silastic $^{\circledR}(8 \mathrm{Fr})$ a través del lumen del conducto, exteriorizados por el hígado y la pared abdominal con la punta distal en el lumen intestinal. En algunos otros casos, estos tutores solo se pusieron a través de la anastomosis sin exteriorizarse al hígado ni al asa de yeyuno. El asa de yeyuno se anastomosó de forma latero-terminal al yeyuno de $40-60 \mathrm{~cm}$ de la hepaticoyeyunostomía y a distancia similar del ligamento de Treitz de manera estándar. Se colocaron dos drenajes cerrados en el sitio de la anastomosis, los cuales se mantuvieron hasta que se documentó ausencia de fuga biliar posterior a la ingestión oral del paciente. En el caso de los tutores transhepáticos, estos fueron colocados a drenaje externo hasta la ausencia de fuga biliar posterior a la ingestión oral del paciente. Estos fueron cerrados y se retiraron un mes después.

\section{Seguimiento y resultados}

Se registraron las complicaciones intrahospitalarias, los días de estancia y la mortalidad intrahospitalaria. Los pacientes fueron seguidos en la consulta externa y estratificados de acuerdo con la clasificación de McDonald para los resultados de las reconstrucciones de LVB $^{27}$. Esta clasificación utiliza las pruebas de función hepática, los síntomas clínicos y los procedimientos invasivos. Los grados A y B se consideran como un tratamiento satisfactorio. Para fines de análisis, se dividió el seguimiento de los pacientes en: 1) pacientes que tuvieran menos de 1 año de seguimiento; 2) pacientes entre 1 y 5 años de seguimiento; y 3) pacientes con más de 5 años de seguimiento. También se dividió de acuerdo con la mediana del seguimiento del paciente y por etapas: desde el inicio del estudio hasta septiembre de 2013 y desde septiembre de 2013 hasta el último paciente incluido en el estudio.

\section{Análisis estadístico}

Las variables continuas se describen como media, desviación estándar y rango, y las variables categóricas como frecuencias y porcentajes. Para identificar los factores asociados a las complicaciones biliares y a la falla terapéutica se realizó un análisis univariado mediante t de Student o U de Mann-Whitney si las variables continuas eran paramétricas o no paramétricas, respectivamente. Se utilizaron la prueba de ji al cuadrado o la prueba exacta de Fisher, o ambas, para el análisis univariado de las variables categóricas. Se realizó un análisis multivariado mediante regresión logística binaria de las variables que fueron estadísticamente significativas en el análisis univariado. Se consideró un valor de $p<0.05$ como estadísticamente significativo. Para el análisis estadístico se utilizó el software SPSS versión 21.

\section{Resultados}

\section{Características y presentación de los pacientes}

Se incluyeron en el estudio 58 pacientes. La edad media fue de $41.8 \pm 14.7$ años (rango: $14-79$ años). 
Fueron de sexo femenino 42 (72.4\%) pacientes y de sexo masculino 16 (27.6\%) pacientes. La CC fue más común (37 pacientes, 63.8\%) que la CL (21 pacientes, $36.2 \%)$. La mayoría de las LVB $(46,79.3 \%)$ fueron diagnosticadas en el posoperatorio. Tras la colecistectomía inicial fueron sometidos a una segunda cirugía $17(29.3 \%)$ pacientes, y 8 (13.8\%) pacientes a dos cirugías. Cuatro $(6.9 \%)$ pacientes tuvieron una reconstrucción de su LVB previa a su referencia; el tiempo promedio de la referencia fue de $9.1 \pm 33$ semanas (rango: 0-250 semanas), con una mediana de 3 semanas. Diez y seis $(27.6 \%)$ pacientes se sometieron a la reconstrucción de la LVB de forma temprana $(<1$ semana) tras haberla sufrido, y $45(77.6 \%)$ pacientes de forma intermedia ( $<6$ semanas). Treinta y siete $(63.8 \%)$ pacientes se sometieron a la reconstrucción de la LVB en la misma admisión de la cirugía inicial.

Los exámenes de laboratorio al ingreso demostraron una hemoglobina media de $11.2 \pm 2 \mathrm{~g} / \mathrm{dl}$ (rango: 6.9$16.1 \mathrm{~g} / \mathrm{dl})$, con un promedio de leucocitos de $10.6 \pm 4.5$ células $/ \mathrm{mm}^{3}$ (rango: 3.2-25.2 células $/ \mathrm{mm}^{3}$ ). Tuvieron ictericia $21(36.2 \%)$ pacientes con bilirrubina sérica total $>2.5 \mathrm{mg} / \mathrm{dl}$, con un promedio de $3.8 \pm 5.1 \mathrm{mg} / \mathrm{dl}$ (rango: 0.2-21.5 mg/dl). La albúmina sérica promedio fue de 3.2 $\pm 0.4 \mathrm{~g} / \mathrm{dl}$ (rango: $2.1-4.4 \mathrm{~g} / \mathrm{dl})$, y $22(37.9 \%)$ pacientes tuvieron una albúmina sérica $<3 \mathrm{~g} / \mathrm{dl}$ en el momento de su referencia. La fosfatasa alcalina promedio fue de $327 \pm 258 \mathrm{UI} / \mathrm{I}$ (rango: 37-1036 UI/I), y 46 (79.2\%) pacientes tuvieron una fosfatasa alcalina superior al rango normal de nuestro laboratorio (129 UI/I).

Once pacientes (19\%) se clasificaron como lesión de tipo B según la clasificación de Amsterdam, 15 (25.9\%) pacientes como tipo C y 32 (55.2\%) pacientes como tipo D. Todos tuvieron lesiones de tipo $\mathrm{E}$ de la clasificación Bismuth-Strasberg: 6 (10.3\%) de tipo E1, 18 (31\%) de tipo E2, $12(20.7 \%)$ de tipo E3, $20(34.5 \%)$ de tipo E4 y $2(3.4 \%)$ de tipo E5. Treinta y cuatro $(58.6 \%)$ pacientes tuvieron lesiones altas (E3-E5) y 20 (34.5\%) perdieron la confluencia de los conductos hepáticos.

\section{Cirugía y resultados}

En 51 (88\%) pacientes se realizó la reconstrucción mediante una hepaticoyeyunostomía de forma término-lateral. Cinco pacientes (8.6\%) tuvieron dos anastomosis hepáticas separadas y 2 (3.4\%) pacientes se sometieron a un hepaticoyeyunostomía intrahepática. En 27 (46.6\%) de las reconstrucciones se utilizaron tutores, de los cuales 11 (19\%) fueron transhepáticos. Los días de estancia hospitalaria promedio fueron $25.2 \pm 18.4$ (rango: 6-100 días).
Tabla 2. Clasificación de McDonald para resultados a largo plazo posterior a una reconstrucción de lesión de vía biliar

\begin{tabular}{llc}
\hline Grado & Descripción & $\mathbf{n}(\%)$ \\
\hline A & $\begin{array}{l}\text { Pruebas de función hepática normales } \\
\text { - Sin síntomas }\end{array}$ & $35(60.3)$ \\
B & $\begin{array}{l}\text { Pruebas de función hepática } \\
\text { ligeramente alteradas - Sin síntomas }\end{array}$ & $12(20.7)$ \\
C & $\begin{array}{l}\text { Pruebas de función hepática anormales } \\
\text { - Colangitis y dolor }\end{array}$ & $6(10.3)$ \\
D & $\begin{array}{l}\text { Revisión endóscopica o quirúrgica } \\
\text { (muerte perioperatoria) }\end{array}$ & $5(8.6)$ \\
\hline
\end{tabular}

Veintitrés (39.6\%) pacientes sufrieron por lo menos una complicación intrahospitalaria. Las complicaciones se dividieron en no biliares y biliares. La complicación no biliar más común fue la infección de herida, en $10(17.2 \%)$ pacientes, seguida de la fistula enterocutánea en $4(6.8 \%)$ pacientes y de la hernia posincisional también en cuatro $(6.8 \%)$ pacientes. Solo $1(1.7 \%)$ paciente desarrolló neumonía y otro tuvo falla hepática, que tuvieron desenlaces fatales (mortalidad del $3.2 \%)$.

La complicación biliar intrahospitalaria más común fue la colangitis en 11 (19\%) pacientes, seguida de la fuga biliar en $6(10.3 \%)$ pacientes. Cuatro (6.9\%) pacientes desarrollaron estenosis posoperatoria (al mes $2,12,18$ y 70 , respectivamente) y 7 (12.1\%) presentaron colangitis recurrente (más de dos episodios durante el seguimiento).

\section{Seguimiento}

Cincuenta y seis pacientes fueron seguidos a través de la consulta externa durante un promedio de 29.8 \pm 27.3 meses (mediana: 23 meses; rango: $1-96$ meses). De los cuatro (6.9\%) pacientes que desarrollaron estenosis, dos se sometieron a una cirugía de revisión de la reconstrucción y los otros dos a colocación de tutores intrahepáticos percutáneos. Dos (3.2\%) pacientes desarrollaron cirrosis hepática. El grado de éxito fue estadificado, de acuerdo con la clasificación de McDonald, en grado A y B, en 47 pacientes (81\%) (Tabla 2). Veintisiete $(48.2 \%)$ pacientes tuvieron un seguimiento entre 1 y 5 años, 21 (37.5\%) pacientes tuvieron un seguimiento < 1 año y $8(14.2 \%)$ pacientes tuvieron un seguimiento $>5$ años. No hubo diferencias significativas entre los pacientes que se operaron antes de septiembre de 2013 y los que se operaron después en cuanto a complicaciones biliares y al éxito terapéutico. Los pacientes con un seguimiento $>5$ 
Tabla 3. Resultados de las complicaciones biliares a largo plazo y del éxito de las reconstrucciones de las lesiones de vía biliar de acuerdo con la clasificación de McDonald

\begin{tabular}{|c|c|c|c|c|c|c|}
\hline Tiempo de seguimiento & Estenosis (n) \% & $p$ & Colangitis recurrente $(\mathrm{n}) \%$ & $p$ & Éxito (McDonald A/B) (n) \% & $p$ \\
\hline \multicolumn{7}{|l|}{ Fecha de la cirugía } \\
\hline Antes de 09/2013 $(n=28)$ & (2) 7.1 & & (5) 17.8 & & (22) 78.5 & \\
\hline Después de 09/2013 ( $n=28)$ & (2) 7.1 & 0.5 & (1) 3.6 & 0.09 & (26) 85.7 & 0.1 \\
\hline \multicolumn{7}{|l|}{ Tiempo de seguimiento } \\
\hline$<1$ año $(n=21)$ & (1) 4.7 & & (0) 0 & & (21) 100 & \\
\hline $1-5$ años $(n=27)$ & (2) 14.8 & & (1)7.4 & & (23) 85.2 & \\
\hline$>5$ años $(n=8)$ & (1) 12.5 & 0.1 & (5) 50 & $0.001^{*}$ & (4) 50 & $0.003^{*}$ \\
\hline \multicolumn{7}{|l|}{ Seguimiento promedio (mediana) } \\
\hline$<23$ meses $(n=27)$ & $(2) 7.4$ & & (0) 0 & & (26) 96.3 & \\
\hline > 23 meses $(n=29)$ & (2) 6.8 & 0.1 & (6) 20.7 & $0.001^{*}$ & (22) 75.9 & $0.03^{*}$ \\
\hline
\end{tabular}

años tuvieron un mayor índice de colangitis recurrente y un menor éxito terapéutico que aquellos con un seguimiento $<5$ años $(p<0.05)$. Los pacientes con un seguimiento $>23$ meses tuvieron menor éxito terapéutico que aquellos con un seguimiento $<23 \mathrm{me}$ ses $(p<0.05)$. Estos resultados se ejemplifican en la tabla 3 .

\section{Factores de riesgo asociados a complicaciones de las reconstrucciones de las LVB}

Se realizó un análisis univariado y multivariado de aquellos pacientes con seguimiento a largo plazo para lograr identificar posibles factores de riesgo asociados a las complicaciones biliares de las reconstrucciones de LVB. Los pacientes con complicaciones biliares tuvieron valores más bajos de hemoglobina, albúmina y fosfatasa alcalina que aquellos sin complicaciones biliares. Estas diferencias fueron estadísticamente significativas $(p<0.05)$. Las complicaciones biliares se presentaron también con más frecuencia en los pacientes que tuvieron lesiones más altas de acuerdo con la clasificación de Bismuth-Strasberg (E3-E5) y en aquellos en los que se utilizaron tutores anastomóticos. Estas diferencias también fueron estadísticamente significativas $(p<0.05)$ (Tabla 3). El análisis multivariado de regresión logística no mostró diferencias estadísticamente significativas entre las variables estadísticamente significativas en el análisis univariado (Tabla 4).

\section{Factores de riesgo asociados al fracaso de las reconstrucciones de LVB}

Se realizó un análisis univariado y multivariado de aquellos pacientes con seguimiento a largo plazo para lograr identificar posibles factores de riesgo asociados al éxito o a la falla terapéutica de las reconstrucciones de LVB. El análisis univariado mostró que los pacientes con éxito terapéutico (McDonald grado A y B) fueron aquellos que no tuvieron un intento de reconstrucción previa, aquellos que se sometieron a una reconstrucción temprana $(<1$ semana) de la LVB y aquellos en los que no se utilizaron tutores. Estas diferencias fueron estadísticamente significativas $(p<0.05)$ (Tabla 5). El análisis multivariado de regresión logística confirmó que la reparación previa y el uso de tutores fueron factores de riesgo en la falla terapéutica de las reconstrucciones de las LVB $(p<0.05)$. La reconstrucción temprana de la LVB tuvo una tendencia hacia la significancia estadística, sin llegar a ella (Tabla 4).

\section{Discusión}

Los resultados de este estudio indican que nuestro centro tiene una buena tasa de éxito en la reconstrucción de las LVB, aunque este éxito disminuye conforme el tiempo de seguimiento se hace superior a 5 años. Además, encontramos que existen factores de riesgo relacionados con las complicaciones y con el éxito de la reconstrucción, tales como el estado general del paciente, el tipo de lesión, el uso de tutores anastomóticos, el tiempo de reconstrucción de la lesión y los intentos previos de reconstrucción antes de la referencia a nuestra unidad.

El objetivo principal de las reconstrucciones de LVB es lograr una adecuada excreción biliar sin causar sintomatología postoperatoria ni trastornos de la función hepática. Desde las publicaciones de Lillemoe, et al. ${ }^{6}$ y de Sicklick, et al. ${ }^{7}$, en las que el éxito de las reconstrucciones de LVB (categorizadas como asintomáticas con pruebas de función hepática normales 
Tabla 4. Análisis univariado/multivariado de los factores asociados a complicaciones biliares en la reconstrucción de lesiones de vía biliar

\begin{tabular}{|c|c|c|c|c|}
\hline \multirow[t]{2}{*}{ Factores } & Univariado & \multirow[t]{2}{*}{$\mathrm{p}$} & Multivariado & \multirow[t]{2}{*}{ p } \\
\hline & Media \pm DE Razón momios (IC 95\%) & & Exp (B) Razón momios (IC 95\%) & \\
\hline \multicolumn{5}{|l|}{ Hemoglobina (g/dl) } \\
\hline Complicación & $10.4 \pm 1.6$ & $0.03^{*}$ & $0.04(-0.04-0.13)$ & 0.3 \\
\hline No complicación & $11.6 \pm 2.0$ & & & \\
\hline \multicolumn{5}{|l|}{ Albumina (g/dl) } \\
\hline Complicación & $2.9 \pm 0.5$ & $0.04^{*}$ & $0.1(-0.42-0.46)$ & 0.9 \\
\hline No complicación & $3.4 \pm 0.6$ & & & \\
\hline Albumina $<3 \mathrm{~g} / \mathrm{dl}$ & $3.06(1.1-8.3)$ & $0.02^{\ddagger}$ & $0.2(-0.27-0.79)$ & 0.3 \\
\hline \multicolumn{5}{|l|}{ Fosfatasa alcalina (UI/I) } \\
\hline Complicación & $209.2 \pm 130$ & $0.009^{\dagger}$ & $0(-0.001-0.001)$ & 0.6 \\
\hline No complicación & $406.3 \pm 292$ & & & \\
\hline Fosfatasa alcalina $<129 \mathrm{IU} / \mathrm{I}$ & $2.1(0.81-5.43)$ & $0.04^{\ddagger}$ & $-0.2(-0.68-0.2)$ & 0.2 \\
\hline Lesiones Bismuth Strasberg III-V & $1.4(1.04-2.11)$ & $0.03^{\ddagger}$ & $-0.06(-0.4-0.27)$ & 0.6 \\
\hline Tutores transanastomóticos & $3.2(1.32-7.82)$ & $0.004^{\ddagger}$ & $0.33(-0.046-0.71)$ & 0.08 \\
\hline
\end{tabular}

*Estadísticamente significativo por t de Student.

tEstadísticamente significativo por $U$ de Mann-Whitney.

ثEstadísticamente significativo por ji al cuadrado.

IC: intervalo de confianza.

Tabla 5. Análisis univariado/multivariado de los factores asociados a la falla terapéutica de las reconstrucciones de lesiones de vía biliar

\begin{tabular}{|c|c|c|c|c|}
\hline \multirow[t]{2}{*}{ Factores } & Univariado & \multirow[t]{2}{*}{ p } & Multivariado & \multirow[t]{2}{*}{ p } \\
\hline & Razón momios (IC 95\%) & & Exp (B) Razón momios (IC 95\%) & \\
\hline Reconstrucción previa (no) & $6.5(2.5-16.6)$ & $0.01^{*}$ & $-0.503(-0.834-0.171)$ & $0.004^{\dagger}$ \\
\hline Reconstrucción en > 1 semana & $1.329(1.09-1.52)$ & $0.036^{+}$ & $0.172(-0.015-0.358)$ & 0.07 \\
\hline Tutores transanastomóticos (no) & $9.9(1.32-74.1)$ & $0.005^{\star}$ & $-0.242(-0.411-0.073)$ & $0.006^{+}$ \\
\hline
\end{tabular}

*Estadísticamente significativo por prueba exacta de Fisher

†Estadísticamente significativo por regresión logística binaria.

IC: intervalo de confianza.

o ligeramente alteradas) fue de un $90.8 \%$, el éxito terapéutico ha sido descrito en un rango que va del $75^{9}$ al $93 \%^{11}$, con cifras intermedias ${ }^{15}$. La eficacia y la seguridad de la reconstrucción de LVB incluye morbilidad de origen biliar y morbilidad en general. La tasa de complicaciones de las reconstrucciones de LVB es del 15 al 43\%, 7,,13,16,18 en centros hepatobiliares especializados, y aumenta hasta casi el $75 \%$ en hospitales donde no hay especialistas hepatobiliares ${ }^{16}$. Se ha descrito una incidencia de fuga biliar posoperatoria del $5 \%$, colangitis (posoperatoria o recurrente) en un $5-27 \%$ y estenosis bilioentérica en un $5-10 \%^{7,12}$. Algunos centros han logrado disminuir la incidencia de colangitis y de estenosis del 11 al 6\% y del 13 al 5\%, respectivamente, al crecer como referencia y obtener más experiencia en el manejo de estos $\operatorname{casos}^{18}$; y exceptuando algunos casos de mortalidad superior al $6 \%{ }^{11}$, esta es cercana al $2 \% \%^{7,8}$.
Nuestro centro ha conseguido obtener una buena tasa de éxito, del $81 \%$, de acuerdo con la clasificación de McDonald durante todo el seguimiento, similar y superior al $75 \%$ de acuerdo con la época de la cirugía realizada (antes o después de 2013) y en los pacientes con seguimiento mayor o menor de 2 años aproximadamente (76 vs. 93\%), pese a que esta diferencia sea estadísticamente significativa. También hemos sido capaces de lograr una eficacia y una seguridad adecuadas (morbilidad del 39\%: 12-19\% colangitis (posoperatoria y recurrente), $10 \%$ fuga biliar y $6 \%$ estenosis, con una mortalidad del $3 \%$ en una pléyade de pacientes con lesiones complejas (grado B y D de Amsterdam) y secciones o estenosis del hilio biliar altas (lesiones E3-E5 de Bismuth-Strasberg). Tanto la estenosis como la colangitis recurrente permanecieron en porcentajes eficaces y seguros de acuerdo con los periodos de tiempo en seguimiento, excepto la 
incidencia de colangitis en los pacientes que fueron seguidos durante más de 5 años. El seguimiento prolongado de las reconstrucciones de LVB permite evaluar la ausencia de estenosis biliar (objetivo de éxito en estos casos). La estenosis puede ocurrir a diferentes intervalos del procedimiento, hasta 7 años después de su realización $n^{5,7}$.Algunos autores tienen como meta de seguimiento adecuado entre 2 y 5 años ${ }^{14}$, y otros lo extienden hasta 20 años ${ }^{20}$. Por ello, nosotros seguimos a nuestros pacientes la mayor cantidad de tiempo posible para asegurarnos de que se mantiene el éxito terapéutico. Así mismo, el seguimiento a largo plazo de los pacientes permite establecer curvas de crecimiento y experiencia, como ha sido descrito en programas que han logrado un aumento en su referencia ${ }^{8}$, y en casos seleccionados poder evaluar la evaluación del daño hepático que pudiera existir previo a la reconstrucción ${ }^{22}$.

A pesar de ser una técnica estandarizada, nosotros encontramos que el uso de tutores en la anastomosis hepática (transhepáticos o no) fue un factor de riesgo asociado a las complicaciones biliares en el análisis univariado, y a falla terapéutica tanto en el análisis univariado como en el multivariado. Para su uso, seguimos las recomendaciones previamente publicadas $^{15,22}$ (tejido isquémico o con fibrosis cicatricial y conductos de diámetro $<4 \mathrm{~mm}$ ), y al igual que otros, las complicaciones son menores cuando no se utilizan.

Los especialistas hepatobiliares han tratado de identificar posibles factores de riesgo relacionados con las complicaciones o con resultados insatisfactorios después de una reconstrucción de LVB. Diferentes estudios han descrito que las reconstrucciones no realizadas por cirujanos hepatobiliares aumentan el riesgo de complicaciones generales y biliares, además de falla terapeutica ${ }^{11,16,17,20,22}$. Las lesiones altas también se han asociado a complicaciones, especialmente a colangitis $11,12,17$. Nosotros encontramos resultados similares con respecto al nivel de la lesión y al uso de tutores, como ya se ha mencionado, además de otros factores como unos valores preoperatorios bajos de albúmina, hemoglobina y fosfatasa alcalina. Aunque la hipoalbuminemia ya había sido referida como un factor asociado a las complicaciones de la reconstrucción de LVB ${ }^{28}$, los valores bajos de hemoglobina y de fosfatasa alcalina no se han relacionado con complicaciones posteriores a LVB. Podríamos sugerir a la hemoglobina como un marcador subrogado de sepsis e inflamación, condiciones que deben evitarse para una reconstrucción de LVB exitosa ${ }^{10,14,15,17,22,28 ;}$ sin embargo, no encontramos relación con los valores de fosfatasa alcalina en el desarrollo de complicaciones. Cabe mencionar que ninguna de estas asociaciones tuvo significancia estadística en el análisis multivariado, de modo similar a lo observado en otros estudios $^{11,13}$.

Además de intentar predecir las complicaciones, es de mayor relevancia poder predecir el éxito en el seguimiento a largo plazo de la reconstrucción de LVB. Encontramos que una reconstrucción previa a la referencia a nuestro centro y el uso de tutores se asocia y es factor de riesgo de falla terapéutica, y que la reconstrucción temprana se asocia al éxito en el tratamiento. El tiempo de la reconstrucción ha sido motivo de debate, ya que algunos sugieren que la reconstrucción debería ser tardía cuando se realiza por cirujanos hepatobiliares ${ }^{11,12}$ y otros describen que el tiempo de la reconstrucción importa si lo hacen cirujanos no especialistas hepatobiliares ${ }^{17}$. Además, una reconstrucción tardía puede acompañarse de lesiones vasculobiliares y procedimientos endoscópicos previos que ensombrecen el éxito del tratamiento ${ }^{21}$. Esta combinación de factores apoya el hecho de que una reconstrucción previa, en especial si no se hizo en un centro de referencia, tiene mayor riesgo de falla terapéutica ${ }^{13,22}$. La asociación de los tutores no solo a las complicaciones, sino también al éxito, justifica su uso selectivo ${ }^{15,22}$.

Aunque nuestro estudio tiene buenos resultados posoperatorios y confirma factores de riesgo para falla terapéutica en las reconstrucciones de LVB, debemos reconocer que los hallazgos descritos surgen de una serie retrospectiva con una muestra no muy grande, y que aún no hemos estandarizado los estudios de imagenología biliar y la evaluación vascular biliar, como se menciona en los métodos, por lo que estas ausencias pudieran afectar de modo negativo nuestro estudio.

En resumen, la reconstrucción de las LVB debe hacerse de forma tal que resulte eficaz y segura. Existen posibles factores de riesgo asociados a la falla en la reconstrucción, como son la reconstrucción previa fuera de un centro de referencia, el uso de tutores anastomóticos y la reconstrucción que no se realiza de forma temprana. Algunos factores perioperatorios, como los valores de hemoglobina, albúmina y fosfatasa alcalina, además del nivel de la lesión y del uso de tutores, pueden asociarse a las complicaciones biliares de estas reconstrucciones. Estos hallazgos deberán ser confirmados al tener una mayor 
referencia de esta patología a nuestro centro y con un seguimiento a más largo plazo.

\section{Conflicto de intereses}

Ninguno que declarar.

\section{Financiamiento}

Ninguno.

\section{Bibliografía}

1. Gouma DJ, Go PM. Bile duct injury during laparoscopic cholecystectomy and conventional cholecystectomy. J Am Coll Surg. 1994;178:229-33.

2. Massarweh NN, Devlin A, Symons RG, Broeckel JA, Flum DR. Risk tolerance and bile duct injury: surgeon characteristics, risk-taking preference, and common bile duct injuries. J Am Coll Surg. 2009;209:17-24.

3. Tantia O, Jain M, Khanna S, Sen B. latrogenic biliary injury: 13,305 cholecystectomies experienced by a single surgical team over more than 13 years. Surg Endosc. 2008;22:1077-86.

4. Flum DR, Cheadle A, Prela C, Dellingwer EP, Chan L. Bile duct injury during cholecystectomy and survival in Medicare beneficiaries. JAMA. 2003;290:2168-73.

5. Ejaz A, Spolverato G, Kim Y, Dodson R, Sicklick JK, Pitt HA, et al. Longterm health-related quality of life after iatrogenic bile duct injury repair. J Am Coll Surg. 2014;5:923-32.

6. Lillemoe KD, Melton GB, Cameron JL, Pitt HA, Campbell KA, Talamini MA, et al. Postoperative bile duct strictures: management and outcome in the 1990s. Ann Surg. 2000;232:430-41.

7. Sicklick JK, Camp MS, Lillemoe KD, Melton GB, Yeo CJ, Campbell KA, et al. Surgical management of bile duct injuries sustained during laparoscopic cholecystectomy. Perioperative results in 200 patients. Ann Surg. 2005;241:786-95

8. Mercado MA, Franssen B, Domínguez I, Arriola JC, Ramírez F, Elnecavé A, et al. Transition from a low-to a high-volume centre for bile duct repair: changes in technique and improved outcome. HPB. 2011;13:767-73.

9. Nuzzo G, Giuliante F, Giovannini I, Murazio M, D'Acapito F, Ardito F, et al. Advantages of multidisciplinary management of bile duct injuries during cholecystectomy. Am J Surg. 2008;195:763-9.

10. Stewart L, Way LW. Laparoscopic bile duct injuries: timing of surgical repair does not influence success rate. A multivariate analysis of factors influencing surgical outcomes. HPB. 2009;11:516-22.

11. Schmidt SC, Langrehr JM, Hintze RE, Neuhaus P. Long-term results and risk factors influencing outcome of major bile duct injuries following cholecystectomy. Br J Surg. 2005;92:76-82.
12. Walsh RM, Henderson JM, Vogt DP, Brown N. Long-term outcome of biliary reconstruction for bile duct injuries from laparoscopic cholecystectomies. Surgery. 2007;142:450-7.

13. Pottakkat B, Vijayahari R, Prakash A, Singh RK, Behari A, Kapoor VK, et al. Factors predicting failure following high bilio-enteric anastomosis for post-cholecystectomy benign biliary strictures. J Gastrointest Surg. 2010;14:1389-94.

14. Huang $\mathrm{Q}$, Yao HH, Shao F, Wang $\mathrm{C}, \mathrm{Hu}$ YG, Hu S, et al. Analysis of risk factors for postoperative complication of repair of bile duct injury after laparoscopic cholecystectomy. Dig Dis Sci. 2014;59:3085-91.

15. Mercado MA, Chan C, Orozco H, Cano G, Chaparro JM, Galindo E, et al To stent or not to stent bilioenteric anastomosis after iatrogenic injury. A dilemma not answered? Arch Surg. 2002;137:60-3.

16. Mercado MA, Vilatoba M, Contreras A, Leal P, Cervantes E, Arriola JC, et al. latrogenic bile duct injury with loss of confluence. World J Gastrointest Surgery. 2015;7:254-60.

17. Perera MT, Silva MA, Hegab B, Muralidharan V, Branhall SR, Mayer AD, et al. Specialist early and intermediate repair of post-laparoscopic cholecystectomy bile duct injuries is associated with an improved long-term outcome. Ann Surg. 2011;253:553-60.

18. Fisher CP, Fahy BN, Aloia TA, Bass BL, Gaber O, Ghobrial RM. Timing of referral impacts surgical outcomes in patients undergoing repair of bile duct injuries. HPB. 2009;11:32-7.

19. DeReuver PR, Grossmann I, Busch OR, Obertop H, van Gulik TM, Gouma DJ. Referral pattern and timing of repair are risk factors for complications after reconstructive surgery for bile duct injury. Ann Surg. 2007;245:763-70.

20. Patrono D, Benvenga R, Colli F, Baroffio P, Romagnoli R, Salizzoni M. Surgical management of post-cholecystectomy bile duct injuries: referral patterns and factors influencing early and long-term outcome. Updates Surg. 2015;67:283-91.

21. Kirks RC, Barnes TE, Lorimer PD, Cochran A, Siddiqui I, Martine JB, et al. Comparing early and delayed repair of common bile duct injury to identify clinical drivers of outcome and morbidity. HPB. 2016; 18:718-25.

22. Domínguez I, Sanford DE, Liu J, Hawkins WG, Mercado MA. Timing of surgical repair after bile duct injuries impact postoperative complications but not anastomotic patency. Ann Surg. 2016;264:544-53.

23. Neuhaus $P$, Schmidt SC, Hintze RE, Adler A, Veltzke W, Raakow R, et al. Classification and treatment of bile duct lesions following laparoscopic cholecystectomy. Chirurg. 2000;71:166-73.

24. Bismuth $H$, Majno PE. Biliary strictures: classification based on the principles of surgical treatment. World J Surg. 2001;25:1241-4.

25. Strasberg S, Hertl M, Soper N. An analysis of the problem of biliary injury during laparoscopic cholecystectomy. J Am Coll Surg. 1995; 180:101-25.

26. Clavien $P$, Sarr MG, Fong Y. Atlas of upper gastrointestinal and hepato-pancreato-biliary surgery. Berlin Heidelberg: Springer-Verlag; 2007.

27. McDonald ML, Farnell MB, Nagorney DM, Ilstrup DM, Kutch JM. Benign biliary strictures: repair and outcome with a contemporary approach. Surgery. 1995;118:582-90.

28. Mercado MA, Chan C, Orozco H, Podgaetz E, Porras DE, De la Medina AR, et al. Low serum albumin is not a contraindication for early iatrogenic bile duct injury repair. Ann Hepatol. 2005;4:184-7. 